\title{
Macrophage-specific transgenic expression of cholesteryl ester hydrolase significantly reduces atherosclerosis and lesion necrosis in Ldlr $\mathrm{r}^{--}$mice
}

\author{
Bin Zhao, ${ }^{1}$ Jingmei Song, ${ }^{1}$ Woon N. Chow, ${ }^{2}$ Richard W. St. Clair, ${ }^{3}$ \\ Lawrence L. Rudel, ${ }^{3}$ and Shobha Ghosh ${ }^{1}$
}

\begin{abstract}
1Department of Internal Medicine and 'Department of Anatomy and Neurobiology, Virginia Commonwealth University, Richmond, Virginia, USA. ${ }^{3}$ Department of Pathology, Lipid Sciences Section, Wake Forest University School of Medicine, Winston-Salem, North Carolina, USA.
\end{abstract}

\begin{abstract}
Accumulation of cholesteryl esters (CEs) in macrophage foam cells, central to atherosclerotic plaque formation, occurs as a result of imbalance between the cholesterol influx and efflux pathways. While the uptake, or influx, of modified lipoproteins is largely unregulated, extracellular acceptor-mediated free cholesterol (FC) efflux is rate limited by the intracellular hydrolysis of CE. We previously identified and cloned a neutral $\mathrm{CE}$ hydrolase (CEH) from human macrophages and demonstrated its role in cellular CE mobilization. In the present study, we examined the hypothesis that macrophage-specific overexpression of CEH in atherosclerosis-susceptible $\mathrm{Ldlr}^{-/-}$mice will result in reduction of diet-induced atherosclerosis. Transgenic mice overexpressing this CEH specifically in the macrophages (driven by scavenger receptor promoter/enhancer) were developed and crossed into the $\mathrm{Ldll}^{-/-}$background (Ldlr ${ }^{-/}$CEHTg mice). Macrophage-specific overexpression of CEH led to a significant reduction in the lesion area and cholesterol content of high-fat, high-cholesterol diet-induced atherosclerotic lesions. The lesions from $\mathrm{Ldll}^{-/-\mathrm{CEHTg}}$ mice did not have increased FC, were less necrotic, and contained significantly higher numbers of viable macrophage foam cells. Higher $\mathrm{CEH}$-mediated FC efflux resulted in enhanced flux of FC from macrophages to gall bladder bile and feces in vivo. These studies demonstrate that by enhancing cholesterol efflux and reverse cholesterol transport, macrophage-specific overexpression of $\mathrm{CEH}$ is antiatherogenic.
\end{abstract}

\section{Introduction}

Presence of cholesteryl ester-laden (CE-laden) macrophage foam cells is one of the most prominent characteristics of an atherosclerotic lesion. Foam cells develop in the vessel wall as a result of migration of circulating monocytes into the intima, differentiation into macrophages, and uptake of excessive amounts of retained and modified plasma lipoproteins. CEs associated with the lipoproteins are hydrolyzed in late endosomes/lysosomes to free cholesterol (FC), which then traffics to and integrates into the plasma membrane (1). Excess membrane cholesterol and a fraction of LDL-derived FC is transported to endoplasmic reticulum where it is reesterified by acyl CoA:cholesterol acyltransferase 1 (ACAT-1) and stored in cytoplasmic lipid droplets. While this reesterification of cholesterol is initially beneficial to the cells in preventing the FC-associated cell toxicity, under conditions of unregulated or increased uptake of modified LDL, it leads to excessive accumulation of CE present as cytoplasmic lipid droplets, giving the cells their characteristic "foamy" appearance. Extracellular acceptor-mediated cholesterol efflux is the primary mechanism for removal of cellular cholesterol and is critical in preventing foam

Nonstandard abbreviations used: ACAT-1, acyl CoA:cholesterol acyltransferase; AcLDL, acetylated LDL; CE, cholesteryl ester; CEH, CE hydrolase; CEL, carboxyl ester lipase; EC, esterified cholesterol; FC, free cholesterol; FPLC, fast protein liquid chromatography; HSL, hormone-sensitive lipase; Ldlr ${ }^{-/}$, LDL receptor-null (mice). Conflict of interest: The authors have declared that no conflict of interest exists. Citation for this article: J. Clin. Invest. 117:2983-2992 (2007). doi:10.1172/JCI30485 cell formation and atherosclerotic lesion development. However, in order for efflux to occur, FC must first be released from stored $\mathrm{CE}$ by hydrolysis mediated by a neutral CE hydrolase (CEH), and this step is increasingly being recognized as the rate-limiting step in FC efflux (2).

Despite the obvious significance of CE hydrolysis in cellular FC efflux, limited efforts have been directed toward manipulating this step as a potential target for pharmacological or genetic intervention of foam cell formation. The main reason had been the controversy surrounding the identity of neutral $\mathrm{CEH}(\mathrm{s})$ in macrophages. Although several lines of evidence initially suggested that the enzyme responsible for CE hydrolysis in murine macrophages is similar to hormone-sensitive lipase (Hsl), which is present in adipose and steroidogenic tissues (3-5), identical levels of CE hydrolysis were observed in peritoneal macrophages isolated from wild-type or Hsl knockout mice $(6,7)$, suggesting that an enzyme other than Hsl is responsible for catalyzing CE hydrolysis in murine macrophages. Further, Hsl-transgenic mice developed 2-3 times larger lesions, and macrophages from these animals stored correspondingly higher concentrations of CE when incubated with acetylated LDL (AcLDL) (8), indicating a limited role, if any, of Hsl in cholesterol removal from macrophages. $\mathrm{Li}$ and Hui reported the absence of Hsl in human macrophages and demonstrated the expression of bile salt-stimulated $\mathrm{CEH}$, similar to secretory pancreatic carboxyl ester lipase (Cel) (9). However, macrophage-specific transgenic expression of this Cel increased $\mathrm{CE}$ accumulation and promoted atherosclerosis (10), confirming 


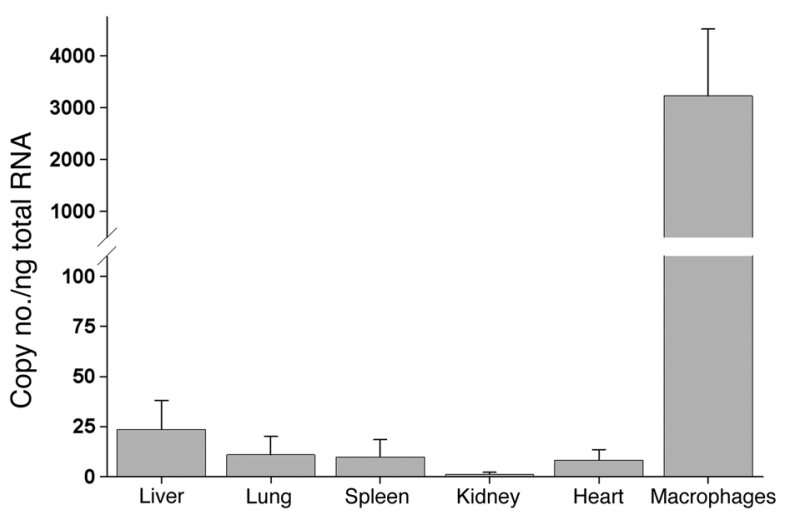

Figure 1

Macrophage-specific expression of the human $\mathrm{CEH}$ transgene. Total RNA was isolated from liver, lung, spleen, kidney, heart, and thioglycollate-elicited peritoneal macrophages. $C E H$ mRNA expression was determined by real time PCR as described in Methods. Data (mean $\pm \mathrm{SD}, n=3$ ) from founder line in C57BL/6 background are expressed as CEH copy no./ng total RNA. Similar results were obtained from the 2 founder lines in Balb-c/C57BL/6 hybrid background that were also tested.

the initial speculation of $\mathrm{Li}$ and Hui that a secretory enzyme such as Cel is unlikely to play a role in intracellular cholesterol metabolism and prevention of foam cell formation (9).

We recently reported the cloning and characterization of a neutral $\mathrm{CEH}$ from human macrophages and demonstrated the expression of the mRNA for this enzyme in the THP1 human monocyte/macrophage cell line, as well as in human peripheral blood monocytes/macrophages (11). This enzyme associated with the surface of lipid droplets in lipid-laden cells and hydrolyzed CE present in lipid droplets (12). Overexpression of this enzyme resulted in mobilization of cellular CE (13) demonstrating its role in regulating cellular $\mathrm{CE}$ accumulation. Further, stable overexpression of this enzyme in the human monocyte/macrophage cell line THP1 resulted in increased FC efflux by all known pathways and decreased cellular CE content (14). In the present study, we developed a macrophage-specific transgenic mouse model expressing this CEH. Results of this study show that increased CEH expression in macrophages significantly reduces high-fat, high-cholesterol diet-induced atherosclerosis in LDL receptor-null ( $\mathrm{Ldlr}^{-/-}$) mice. In addition, the lesions in $\mathrm{Ldll}^{-/-} \mathrm{CEHTg}$ mice did not have an increase in FC, were less necrotic, and contained significantly higher viable macrophage foam cells, indicating that these lesions are not only smaller but are also biologically different.

\section{Results}

Development of CEH-transgenic mice. CEH-transgenic mice were identified by PCR amplification of tail DNA. One founder line in C57BL/ 6 background and 7 in Balb-c/C57BL/ 6 hybrid background were obtained. The transgenic founder line in C57BL/6 background and 2 of 7 founder lines in Balb-c/C57BL/6 hybrid background were analyzed for tissue distribution of CEH. Similar expression profiles were obtained with all 3 lines, and data for the $\mathrm{C} 57 \mathrm{BL} / 6$ founder line is shown in Figure 1. While CEH expression was seen in liver, lung, spleen, kidney, and heart, significantly higher expression was seen in macrophages, consistent with the use of the SR-A promoter/enhancer (15). Consistent with increased CEH mRNA expression, a $64 \%$ increase in CEH activity $(399 \pm 72.5$ versus $656 \pm 67.9 \mathrm{pmol} / \mathrm{h} / \mathrm{mg}, n=3, P<0.05$ ) was associated with thioglycollate-elicited peritoneal macrophages from transgenic founder mice. This increase in the level of increase in CEH activity, which is fairly modest and potentially in the physiological range, significantly reduced cellular CE levels in agmACAT1 and THP1 macrophages $(13,14)$. Since there was no significant difference in $\mathrm{CEH}$ expression between the 3 founder lines tested, the line in pure C57BL/ 6 background was chosen for further studies after crossing into the Ldlr $^{-/-}$background.

Macrophage-specific transgenic expression of CEH did not alter the plasma cholesterol levels. Plasma was obtained from non-fasted $\mathrm{Ldlr}^{-/}$and Ldlr ${ }^{-/-}$CEHTg mice fed a Western type, high-fat, high-cholesterol diet for 16 weeks and was analyzed for total cholesterol and distribution of cholesterol among lipoprotein fractions. As shown in Figure 2 and Table 1, there was no significant difference in the fast protein liquid chromatography (FPLC) profiles, total plasma cholesterol, or the distribution of cholesterol among the plasma lipoproteins, suggesting that macrophage-specific expression of $\mathrm{CEH}$ does not alter plasma or lipoprotein cholesterol concentrations.

Macrophage-specific transgenic expression of $\mathrm{CEH}$ significantly reduced the atherosclerotic lesion area. Atherosclerosis was assessed in mice fed Western type, high-fat, high-cholesterol diet for 16 weeks. No mortality or morbidity was observed in control or transgenic animals. The percent area of aorta covered with atherosclerotic lesions was determined by en face analyses, and Figure $3 \mathrm{~A}$ shows representative images of aortae obtained from $\mathrm{Ldlr}^{-/-}$and $\mathrm{Ldlr}^{-/-} \mathrm{CEHTg}$ mice. While very few lesions were noticeable in the thoracic and abdominal aortae as expected with this mouse model, the aortic arch displayed the most profound lesions. A marked decrease in atherosclerotic lesion area was observed in the aortic arch (Figure 3B) of CEHtransgenic mice. The quantification of the surface area occupied by atherosclerotic lesions is shown in Figure 3C. Compared with $\mathrm{Ldlr}^{-/-}$mice, transgenic expression of CEH in $\mathrm{Ldlr}^{-/} \mathrm{CEHTg}$ mice led to a significant decrease in percent area occupied by atherosclerotic lesions in the aortic arch $(27.5 \pm 2.53$ versus $14.09 \pm 1.18$ in males, $P=0.0008 ; 22.7 \pm 1.94$ versus $11.14 \pm 1.04$ in females, $P=0.004$ ).

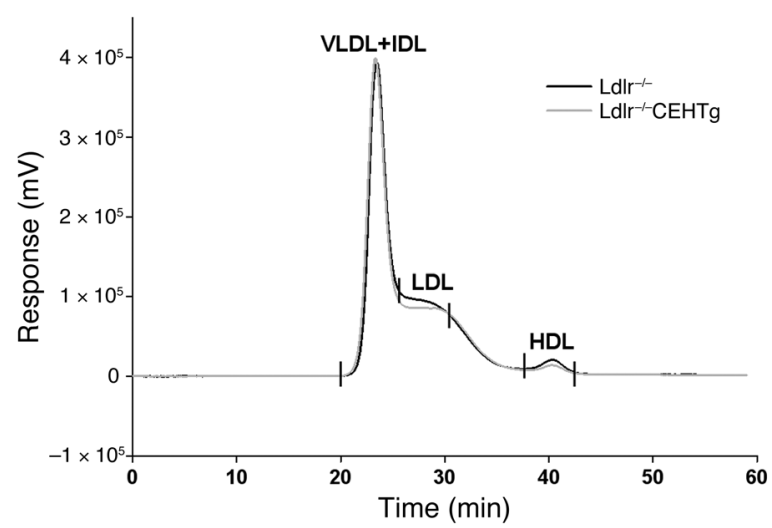

Figure 2

Distribution of cholesterol among plasma lipoprotein fractions. Plasma was obtained from non-fasted $\mathrm{Ldlr}^{-/-}$and $\mathrm{Ldlr}^{-/} \mathrm{CEHTg}$ mice after 16 weeks of feeding. An aliquot of the plasma from each animal was applied to Superose 6 FPLC column (flow rate, $0.4 \mathrm{ml} / \mathrm{min}$ ) and cholesterol content of individual lipoproteins determined by an online assay as described in Methods. A representative FPLC profile is presented showing a continuous online measurement of cholesterol (expressed as millivolts). $n=9$ per genotype. 


\section{Table 1}

Quantification of total cholesterol and its distribution among the different lipoprotein classes

\begin{tabular}{lcccc} 
Genotype & \multicolumn{4}{c}{ Cholesterol (mg/dl, mean \pm SEM) } \\
& Total & VLDL + IDL & LDL & HDL \\
Ldlr $^{--}$ & $2,270 \pm 132$ & $1,309 \pm 85$ & $888 \pm 54$ & $72.7 \pm 3.6$ \\
Ldlr--CEHTg & $1,950 \pm 163$ & $1,090 \pm 110$ & $781 \pm 59$ & $79.2 \pm 3.3$ \\
$P$ value & 0.15 & 0.14 & 0.21 & 0.22 \\
\hline
\end{tabular}

$n=9$ per genotype.

However, there was no significant decrease in the surface area occupied by the lesions in thoracic and abdominal aortae. Total area occupied by the lesions in the entire aorta was calculated by adding the corresponding areas in aortic arch and thoracic and abdominal aortae, and compared with $\mathrm{Ldll}^{-/-}$mice, transgenic expression of $\mathrm{CEH}$ in Ldlr ${ }^{-/}$CEHTg mice led to a significant decrease in total area occupied by the lesions $(16.99 \pm 0.96$ versus $8.69 \pm 0.55$ in males, $P=0.0005 ; 14.21 \pm 1.58$ versus $7.79 \pm 0.66$ in females, $P=0.018$ ). This reduction in the lesion area (about 50\%) by transgenic expression of $\mathrm{CEH}$ was similar and not significantly different in male and female mice. These findings indicate that macrophage-specific $\mathrm{CEH}$ expression decreases diet-induced atherosclerosis in $\mathrm{Ldll}^{-/-}$mice.

Aortic esterified cholesterol content of Ldlr ${ }^{-1-}$ CEHTg mice is significantly lower than that of control Ldlr-/- mice. Aortic cholesterol content is a biochemical endpoint for evaluating atherosclerosis, and a strong positive correlation exists between area occupied by lesions and aortic cholesterol content (16). To evaluate the effect of transgenic expression of $\mathrm{CEH}$ on aortic esterified cholesterol (EC) content, total lipids were extracted from the 2 pieces of the aorta (aortic arch and thoracic and abdominal aortae) and cholesterol content (total cholesterol, FC, and EC) was determined. Consistent with $\mathrm{CEH}$ overexpression and reduced lesion area, the EC content of the aortic arch (Figure 4) was significantly lower (by 50\%) in Ldlr $^{-/-C E H T g ~ m i c e ~ c o m p a r e d ~ w i t h ~} \mathrm{Ldlr}^{-/-}$mice $(116 \pm 8.6$ versus $63 \pm 7.6 \mu \mathrm{g} / \mathrm{mg}$ protein, $P=0.001)$. In addition, the $\mathrm{FC}$ content was also lower in $\mathrm{Ldll}^{-/} \mathrm{CEHTg}$ mice (129 \pm 15.8 versus $60 \pm 7 \mu \mathrm{g} / \mathrm{mg}$ protein, $P=0.009$ ) but the ratio of EC to FC remained essentially unchanged.

\section{Figure 3}

$\mathrm{Ldlr}^{-1-} \mathrm{CEHTg}$ mice have reduced atherosclerosis compared with $\mathrm{Ldll}^{-1-}$ controls. Ldlr ${ }^{-1-}$ and $\mathrm{Ldll}^{-1-} \mathrm{CEHTg}$ mice were fed a Western diet for 16 weeks. Aorta (from aortic sinus to the iliac bifurcation) was isolated, opened and pinned to expose the lesions, and imaged on a black background. (A) Representative images of the aorta. The white horizontal line marks the place where the aortic arch was separated from the thoracic and abdominal aortae. (B) A magnified image of the aortic arch. (C) The quantification of the surface area occupied by the lesions in the aortic arch and the thoracic and abdominal aortae. Data (\% area occupied by the lesions) are expressed as mean \pm SEM for the indicated number of animals in each group. Original magnification, $\times 3 .{ }^{\star} P=0.0008 ;{ }^{* \star} P=0.004$.
In accordance with fewer lesions, cholesterol content of thoracic and abdominal aortae was low, and while the EC and FC content was lower in CEH transgenics compared with $\mathrm{Ldll}^{-/-}$controls, this decrease did not reach statistical significance. Taken together with the en face analyses, these data demonstrate that macrophagespecific expression of $\mathrm{CEH}$ leads to attenuation of diet-induced atherosclerosis in $\mathrm{Ldlr}^{-/-}$mice.

Lesions from Ldlr ${ }^{-1-} \mathrm{CEHTg}$ mice are cellular. Massive accumulation of FC and CE in advanced lesions results in necrotic cores, leading to an acellular and vulnerable plaque. We compared the morphology of the high-fat, high-cholesterol diet-induced aortic sinus lesions in $\mathrm{Ldll}^{-/-}$and Ldlr ${ }^{-/-}$CEHTg mice. Serial sections were stained with Masson's trichrome stain and H\&E and immunostained with antibody to macrophage marker MOMA-2. Representative images in Figure 5 clearly show that the lesions from $\mathrm{Ldlr}^{-/}$mice are largely acellular, containing large cholesterol clefts (Figure 5, A and B), while intact foam cells are present throughout the lesions from Ldlr ${ }^{-/}$CEHTg mice (Figure 5, B and C). The quantification of acellular/necrotic area and number of macrophages and TUNEL-
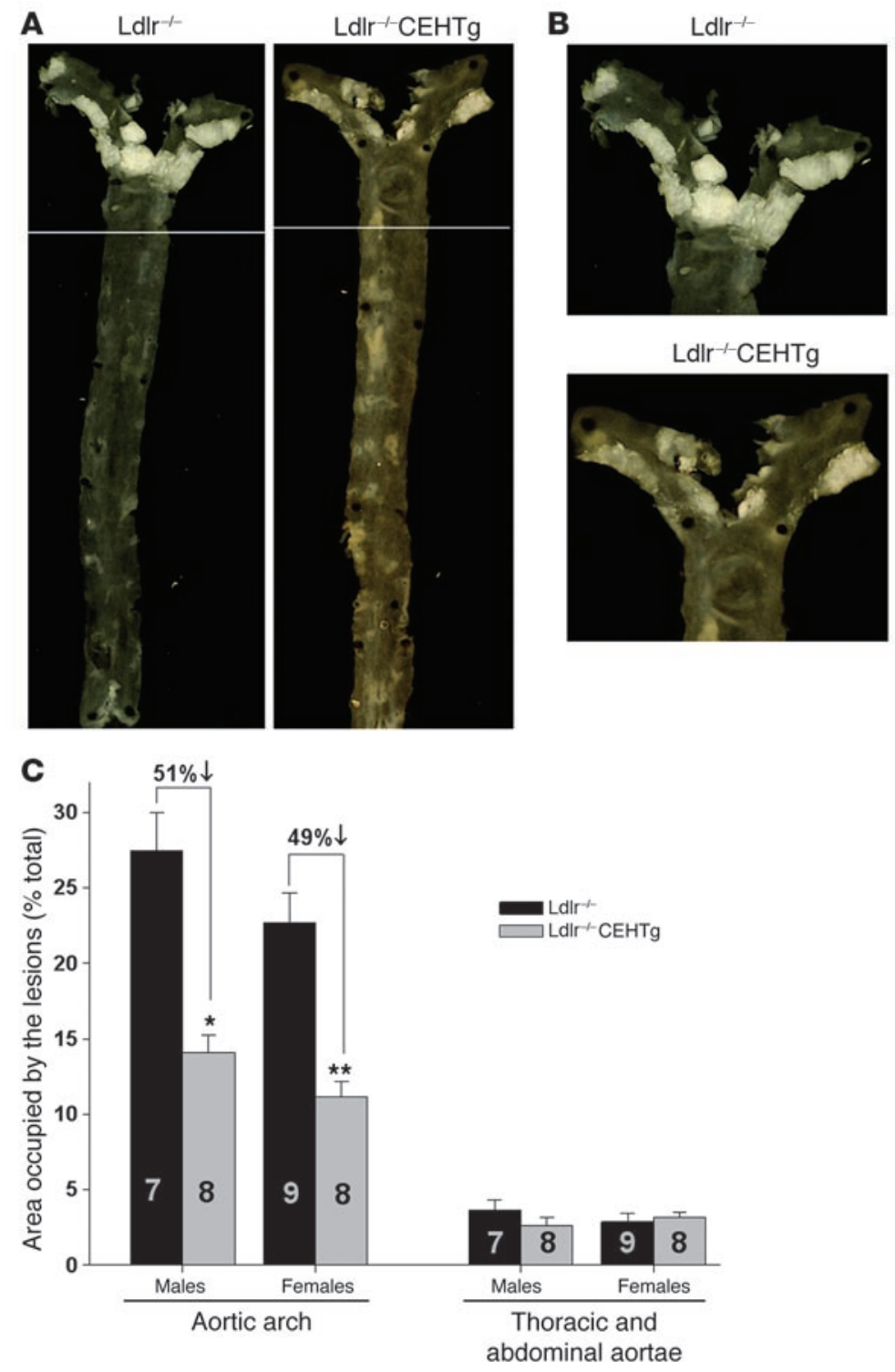


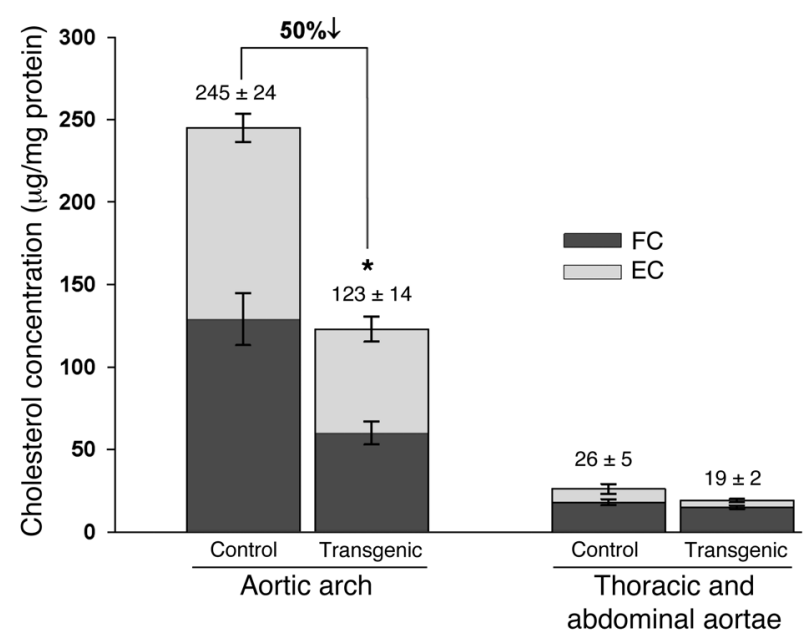

positive macrophage nuclei is shown in Figure 6. The lesions from $\mathrm{Ldlr}^{-/}$- mice were significantly more acellular/necrotic as assessed by the percent necrotic area $(56.79 \pm 14.45$ versus $19.33 \pm 11.33$, $P=0.000004)$. Consistently, the number of macrophages per unit area of the lesion was significantly lower $(21.74 \pm 5.04$ versus $55.87 \pm 22.24, P=0.0007)$. Further, of the number of macrophages present, the percent of TUNEL-positive macrophage nuclei was also significantly higher in the lesions from $\mathrm{Ldll}^{-/-}$mice $(49.48 \pm 27.62$ versus $13.83 \pm 3.64, P=0.0025)$. These data demonstrate that by enhancing intracellular CE hydrolysis and thus facilitating the removal of cholesterol, CEH overexpression prevents cell death and necrosis of lesion-associated foam cells.

Higher FC efflux from peritoneal macrophages isolated from $\mathrm{Ldlr}^{-1}$ CEHTg mice compared with Ldlr ${ }^{-1}$. CEH-mediated hydrolysis of intracellular CEs releases FC for efflux, and this step is considered the rate-limiting step in cholesterol efflux from lipid-laden foam cells. FC is subsequently effluxed by multiple pathways depending on the presence of appropriate exogenous cholesterol acceptors. To determine whether transgenic expression of $\mathrm{CEH}$ increased FC efflux from macrophages, peritoneal macrophages from $\mathrm{Ldlr}^{-/-}$and $\mathrm{Ldlr}^{-/-}$CEHTg mice on chow diet were labeled and loaded with cholesterol by incubating with AcLDL and $\left[{ }^{3} \mathrm{H}\right]$-cholesterol, and FC efflux by the various efflux pathways was monitored. Consistent with our earlier data with CEH overexpression in THP1 macrophages (14), significantly higher efflux

\section{Figure 5}

Lesions in Ldlr ${ }^{--}$CEHTg mice are more cellular. Hearts from Western diet-fed mice were fixed, paraffin embedded, and sectioned as described in Methods. (A) Representative aortic sinus lesion on 1 of the leaflets, stained with Masson's Trichrome reagent. Original magnification, $\times 200$. The lumen (L) of the aortic sinus is so marked for orientation. The red staining of the cytoplasm illustrates the highly cellular nature of the lesion in $\mathrm{Ldll}^{-1-} \mathrm{CEHTg}$ mice. Limited blue staining in the core of the plaque indicates reduced collagen levels in the lesions of $\mathrm{Ldlr}^{-/} \mathrm{com}-$ pared with Ldlr $^{--}$CEHTg mice. (B) Magnification of the boxed areas in A. Serial sections were stained with H\&E. Arrowheads mark the nuclei, arrows point to the cholesterol clefts, and asterisks indicate foam cells. (C) A second serial section was immunostained with antibody to macrophage marker MOMA-2. Intact foam cells (asterisks) containing intracellular lipid droplets and stained brown with MOMA-2 antibody are visible throughout the lesion from $\mathrm{Ldlr}^{-1-} \mathrm{CEHTg}$ but not in lesion from Ldlr $^{-1-}$ mice. Original magnification, $\times 600$ (B and C).

\section{Figure 4}

Aortic lesions in $\mathrm{LdIr}^{-1-} \mathrm{CEHTg}$ mice have significantly lower EC than lesions in Ldlr-1- mice fed a Western diet for 16 weeks. Aortae (from aortic sinus to the iliac bifurcation) were isolated, opened, and pinned to expose the lesions. After fixing in buffered formaldehyde for 24 hours, the aortic arch was cut along the line as shown in Figure 3 and lipids extracted from the resulting 2 pieces corresponding to the arch and the thoracic and abdominal aortae. FC and EC concentration ( $\mu \mathrm{g} / \mathrm{mg}$ protein) was determined as described in Methods. The concentration of total cholesterol is indicated at the top of the bars. Data are expressed as mean $\pm \operatorname{SEM}(n=6)$. ${ }^{\star} P<0.004$.

of $\left[{ }^{3} \mathrm{H}\right]$-cholesterol was observed with macrophages isolated from Ldlr ${ }^{-/-} \mathrm{CEH}$ Tg mice by all efflux pathways, namely aqueous diffusion independent of a cellular transporter (3.04 \pm 0.62 versus $5.12 \pm 0.12, P=0.005)$, ABCA1-mediated efflux to lipid-free ApoA1 $(6.38 \pm 0.75$ versus $11.11 \pm 1.05, P=0.003)$, ABCG1-mediated efflux to $\operatorname{HDL}(24.27 \pm 1.5$ versus $33.54 \pm 0.44, P=0.0004)$, and total efflux to $10 \%$ FBS $(39.22 \pm 2.47$ versus $53.78 \pm 2.07$, $P=0.0014$ ), confirming the role of $\mathrm{CEH}$-mediated hydrolysis in regulating FC efflux. Further, percent efflux of FC from either cell type increased consistent with the efflux capacity of the exogenous acceptor (no acceptor $<$ ApoAI $<$ HDL $<$ FBS). It should be
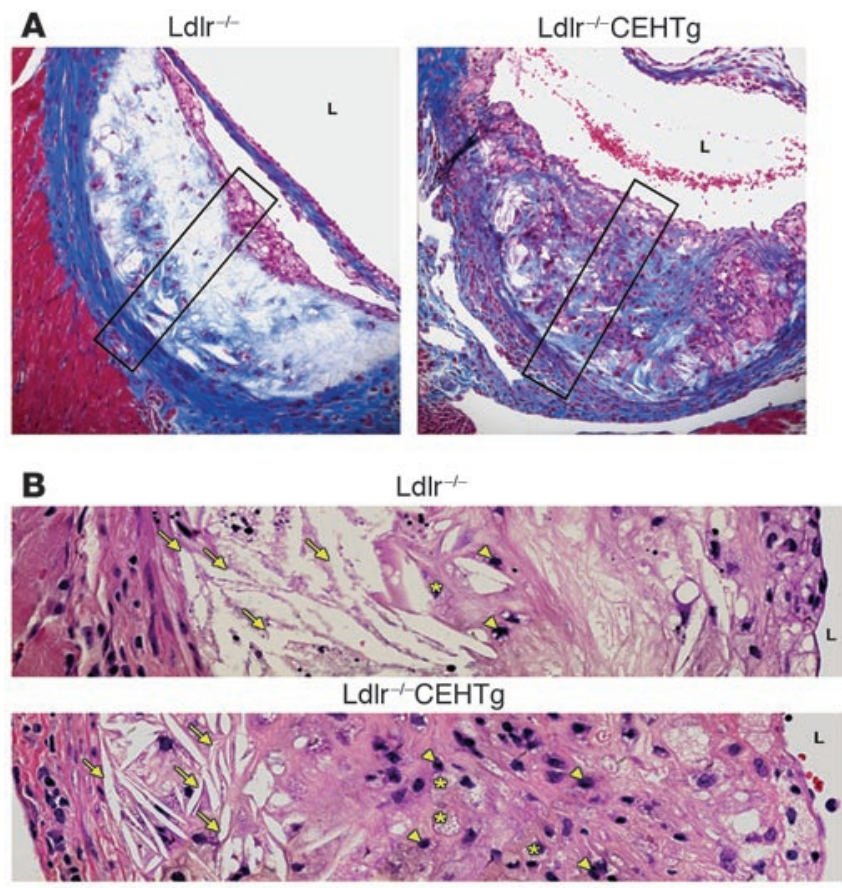

\section{C}
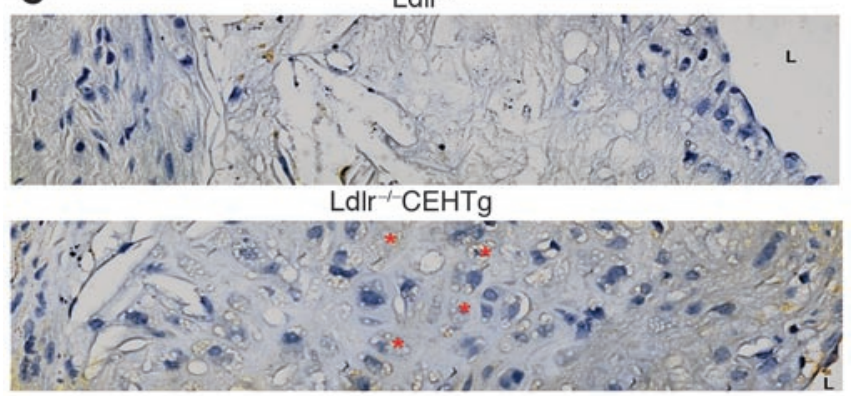

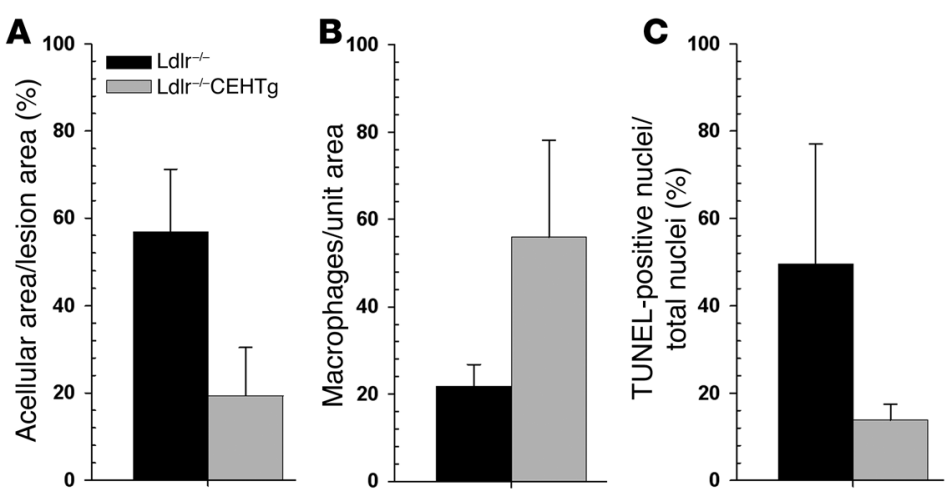

\section{Figure 6}

Decreased necrosis/apoptosis of macrophages in lesions from Ldlr ${ }^{-1-C E H T g ~ c o m p a r e d ~ w i t h ~} \mathrm{Ldll}^{-1-}$ mice. H\&E-stained sections were used to determine the percentage of acellular area/necrotic area of the lesions $(\mathbf{A})$, and the number of macrophages in the lesions (B) as well as the percentage of TUNEL-positive nuclei/total nuclei (C) were determined as described in Methods. Data are expressed as mean \pm SEM for 5 mice per genotype, and 3 lesions were analyzed per animal. $P=0.00002(\mathbf{A}) ; P=0.001(\mathbf{B}) ; P=0.000008(\mathbf{C})$.

noted that FC efflux to HDL and FBS will include the net efflux via SR-BI (17). Taken together with the lesion analysis data, these data are consistent with the conclusion that the $\mathrm{CEH}$-mediated increase in FC efflux from lipid-laden foam cells leads to attenuation of CE accumulation in aortic lesions and reduction in lesion area.

The FC efflux was also monitored from the 2 founder lines obtained in Balb-c/C57BL/6 hybrid background. Consistent with $\mathrm{CEH}$ expression in macrophages of these founders, significantly higher $\left[{ }^{3} \mathrm{H}\right]$-cholesterol efflux was observed with macrophages isolated from transgenic mice compared with nontransgenic littermate controls (data not shown).

Macrophages from $\mathrm{Ldll}^{-1-} \mathrm{CEHTg}$ mice contain lower levels of CE and FC in the presence of exogenous cholesterol acceptor. To determine whether $\mathrm{CEH}$-mediated increased FC efflux led to a reduction in cellular cholesterol content of peritoneal macrophages, EC and FC mass was compared in thioglycollate-elicited peritoneal macrophages isolated from $\mathrm{Ldll}^{-/-}$and $\mathrm{Ldll}^{-/-} \mathrm{CEHTg}$ mice. Following AcLDL loading, cells were incubated either in the absence (serum-free medium) or presence (medium with 10\% serum) of exogenous acceptor for 24 hours. As shown in Figure 7, while there was no significant change in either EC or FC mass in cells incubated in the absence of exogenous acceptor, macrophages from CEHTg mice had significantly lower cellular EC $(67.9 \%$ versus $38.1 \%, P=0.048)$ and FC $(54.8 \%$ versus $16.2 \%, P=0.05)$ mass when cells were incubated in the presence of serum. These data demonstrate that in the presence of an exogenous acceptor, transgenic expression of $\mathrm{CEH}$ levels led to a significantly higher reduction in cellular EC and FC mass.

Similar gene expression in peritoneal macrophages from $\mathrm{Ldlr}^{-/}$and $\mathrm{Ldlr}^{-1-C E H T g}$ mice. To determine whether transgenic expression of
$\mathrm{CEH}$ affects the expression of other genes involved in cholesterol influx (scavenger receptors Cd36 and Msr1) or efflux (cholesterol transporters Abca1, Abcg1, and Scarb1), expression of these genes in nonloaded and AcLDL-loaded peritoneal macrophages from $\mathrm{Ldlr}^{-/-}$and Ldlr ${ }^{-/-}$CEHTg mice was monitored by real-time PCR and data expressed as a percentage of nonloaded control cells (Figure 8). Consistent with earlier studies, there was an increase in the expression of CD36, ABCA1, and ABCG1 in response to lipid loading. However, there was no significant difference in the gene expression between macrophages from $\mathrm{Ldlr}^{-/-}$and $\mathrm{Ldll}^{-/-} \mathrm{CEHTg}$.

Increased expression of $\mathrm{CEH}$ in macrophages results in enhanced reverse cholesterol transport in vivo. To evaluate the effect of transgenic expression of $\mathrm{CEH}$ in macrophages on in vivo reverse cholesterol transport, we employed the model developed by Zhang et al. (18), except that we used mouse peritoneal macrophages isolated from $\mathrm{Ldlr}^{-/-}$or Ldlr ${ }^{-/-}$CEHTg mice instead of the J774 murine macrophage cell line. Ex vivo cholesterol-loaded and -labeled mouse peritoneal macrophages were injected into the peritoneal cavity of chow-fed C57BL/ 6 mice, and movement of $\left[{ }^{3} \mathrm{H}\right]$-cholesterol to gall bladder bile and feces was determined. As shown in Figure 9 , greater than 2 -fold higher $\left[{ }^{3} \mathrm{H}\right]$-cholesterol was associated with bile acids in gall bladder bile $(0.17 \%$ versus $0.56 \%$ of injected, $P=0.0007)$ and feces $(0.62 \%$ versus $1.48 \%$ of injected, $P=0.03)$ when mice were injected with macrophages from $\mathrm{Ldlr}^{-/-C E H T g}$ mice. No significant difference was seen in biliary or fecal $\left[{ }^{3} \mathrm{H}\right]$ cholesterol. The 2-fold higher delivery of macrophage-derived $\left[{ }^{3} \mathrm{H}\right]$-cholesterol to gall bladder bile and feces clearly demonstrates an enhanced reverse cholesterol transport from macrophages isolated from $\mathrm{Ldlr}^{-/-} \mathrm{CEHTg}$ mice.

\section{Figure 7}

Peritoneal macrophages from $\mathrm{CEH}$-transgenic mice have lower EC content. Thioglycollate-elicited peritoneal macrophages from chow-fed mice were isolated and loaded with AcLDL. After a 24-hour equilibration period, 1 set was incubated in serum-free medium (no extracellular acceptor) or in medium containing $10 \%$ FBS as the extracellular acceptor. Lipids were extracted after 24 hours and cellular FC and EC levels determined and normalized to cellular protein. Data are expressed as mean $\pm \mathrm{SD} ; n=3$. The percent decrease in $\mathrm{FC}$ and $\mathrm{EC}$ in the presence of extracellular acceptor is significantly higher in macrophages from Ldll $r^{-1}$ CEHTg mice $\left({ }^{\star} P=0.05\right.$ and $\left.{ }^{* \star} P=0.048\right)$ compared with the corresponding decrease in macrophages from $\mathrm{Ldll}^{-1-}$ mice.

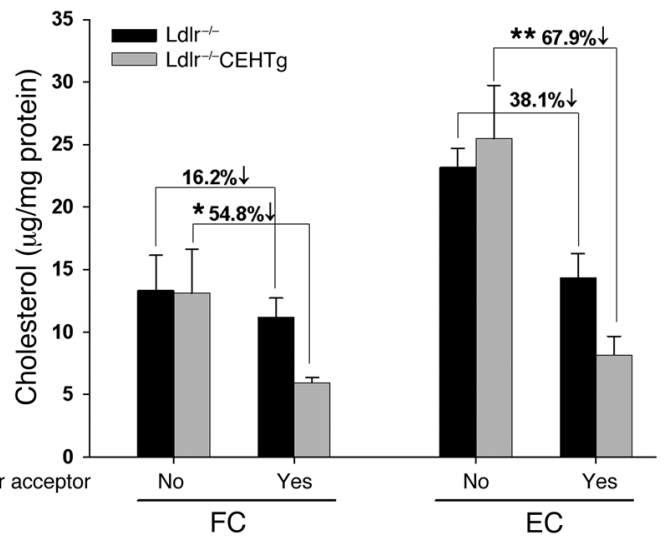




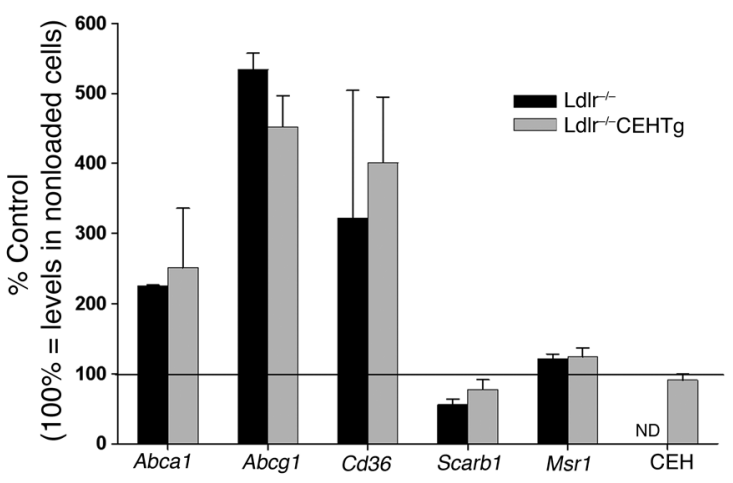

Figure 8

Similar expression of genes involved in cholesterol efflux in peritoneal macrophages isolated from $\mathrm{Ldllr}^{-/-}$and $\mathrm{Ldlr}^{-1-} \mathrm{CEHTg}$ mice. Thioglycollate-elicited peritoneal macrophages from chow-fed mice were isolated and plated in $60-\mathrm{mm}$ culture plates. One set of cells was loaded with AcLDL for 48 hours. Total RNA was isolated from nonloaded as well AcLDL-loaded cells and expression of the indicated genes determined by real time RT-PCR. The level of expression in AcLDL-loaded cells was normalized to that in the corresponding nonloaded cells. Data are expressed as mean $\pm \mathrm{SD} ; n=3$. ND, not detectable.

\section{Discussion}

Extracellular acceptors of cholesterol such as HDL or HDL precursors mediate removal of FC. The importance of this process is underscored by the observed inverse correlation between plasma HDL levels and the risk for coronary heart disease. Since only unesterified cholesterol or FC, and not CE, can efflux from cells, the obligatory first step in the removal of CE from foam cells is the hydrolysis of the intracellular CE. The role for a neutral CEH in hydrolyzing cellular CE was first described by Brown et al. (19) and was further characterized as a cytosolic enzyme in murine macrophages by Khoo and coworkers (20). Based on studies of the kinetics of FC efflux from macrophages, CEH-mediated hydrolysis is now considered to be the rate-limiting step in cellular cholesterol efflux from CE-loaded cells (2). Consistent with this view are the reports of an inverse relationship between $\mathrm{CEH}$ levels and intracellular cholesterol accumulation $(13,21)$. In addition, decreased levels of $\mathrm{CEH}$ expression are observed in macrophages from atherosclerosis-susceptible species $(22,23)$, and overexpression of $\mathrm{CEH}$ results in enhanced mobilization of CE from cells in vitro $(13,14)$.

In order to examine the effects of increased in vivo expression of $\mathrm{CEH}$ on the development of atherosclerosis, we evaluated lesion formation in transgenic mice that overexpress $\mathrm{CEH}$ specifically in macrophages. Our results demonstrate, for what we believe to be the first time, that enhanced expression of $\mathrm{CEH}$ in mouse macrophages leads to a reduction in diet-induced atherosclerosis in $\mathrm{Ldll}^{-/-}$mice (Figure 3). The observed reduction in the aortic atherosclerosis by en face analyses was consistent with the biochemical analyses of the aortic lesions in that the aortic cholesterol content (CE and FC) was also significantly reduced in Ldlr $^{-/}$CEHTg mice (Figure 4).

While modification of cholesterol to enter the bile acid synthesis pathway (24) and direct secretion of cholesterol associated with ApoE secreted by macrophages (25) are 2 additional mechanisms proposed for cholesterol removal, extracellular acceptor-mediated cholesterol efflux is the major pathway for the removal of cellular cholesterol and is critical in preventing foam cell formation and development of atherosclerosis. Multiple pathways exist for the efflux of cellular FC. Efflux of FC via aqueous diffusion that is independent of a cellular transporter occurs with all cell types but is inefficient (26). ABCA1 mediates efflux of both FC and phospholipid to lipid-free ApoAI (22). ABCG1 and ABCG4 stimulate FC efflux to both smaller and larger subclasses of HDLs ( $\mathrm{HDL}_{3}$ and $\mathrm{HDL}_{2}$, respectively) but not to lipid-poor ApoAI (27). SR-BI, on the other hand, mediates bidirectional movement of cholesterol and contributes to the net efflux to HDL and serum (17). Consistent with its role in catalyzing the obligatory first step (CE hydrolysis) in cholesterol efflux and reverse cholesterol transport, transgenic expression of CEH in macrophages from $\mathrm{Ldll}^{-/-} \mathrm{CEHTg}$ mice cells led to an increase in FC efflux to exogenous lipid-free ApoAI (ABCA1-mediated) and to exogenous HDL (ABCG1-mediated). Highest efflux was obtained when $10 \%$ serum was used as the exogenous acceptor, which also contained phospholipids that act as a "sink" for effluxed FC. The data presented here show that while the FC generated by CEH-mediated hydrolysis of CE was effluxed by all known pathways without any apparent preference, the percent FC efflux increase corresponded to the relative importance of these different efflux pathways: aqueous diffusion or transporter independent $<$ ABCA1 mediated $<$ ABCG1 mediated < total efflux (to serum). This enhanced efflux led to a net reduction in FC and EC mass in macrophages from CEHTg mice (Figure 7) in the presence of an extracellular acceptor. Kinetic studies by Mazzone et al. have demonstrated that $90 \%$ of the FC released by CE hydrolysis is available for efflux (as a cholesterol oxidase-accessible pool), but in the absence of an extracellular acceptor it is readily reesterified (28). This is consistent with our data showing a net reduction in macrophage CE content only in the presence of an extracellular acceptor and a significantly greater reduction in cellular CE in macrophages from CEH-transgenic mice (Figure 7).

While extracellular acceptor-mediated efflux of FC from macrophages is the mechanism by which FC is removed from macrophage foam cells, final elimination of cholesterol from the body occurs by the movement of this FC to the liver for excretion as

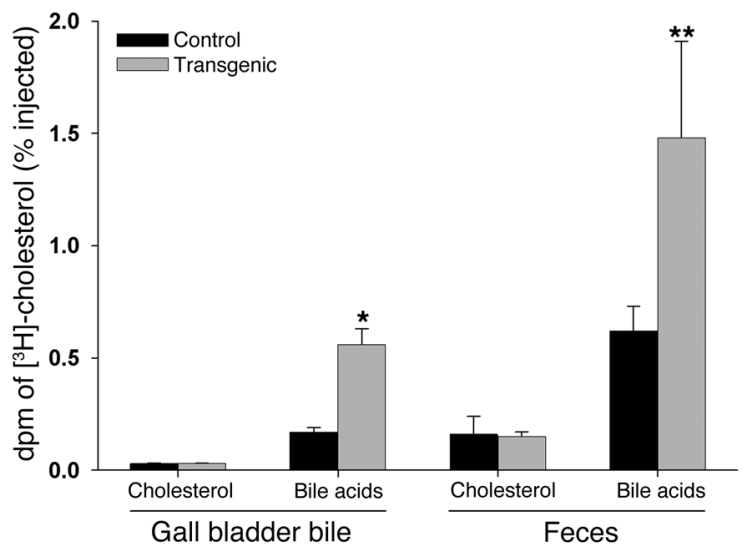

Figure 9

Significantly higher movement of cholesterol from CEHTg macrophages to bile acids. In vivo movement of $\left[{ }^{3} \mathrm{H}\right]$-cholesterol from macrophages to gall bladder bile and feces was monitored as described in Methods. Total radioactivity $(\mathrm{dpm})$ of $\left[{ }^{3} \mathrm{H}\right]$-cholesterol was determined and data (mean $\pm \mathrm{SD} ; n=3$ ) are presented as percentage of the total dpm injected. ${ }^{*} P=0.0007 ;{ }^{* *} P=0.02$. 
bile acids or biliary cholesterol. Our data demonstrate that the movement of $\left[{ }^{3} \mathrm{H}\right]$-cholesterol from macrophage to feces was 2-fold higher with macrophages from $\mathrm{Ldll}^{-/}$CEHTg mice $(1.6 \%$ of injected) compared with those from $\mathrm{Ldlr}^{-/-}$mice ( $0.8 \%$ of injected) and that bile acids were the principal excretory form of $\left[{ }^{3} \mathrm{H}\right]$-cholesterol. Given the equivalent cholesterol content $(4.2-4.4 \mu \mathrm{g})$ of the injected macrophages, this increased movement would lead to increased removal of cholesterol from the body. Thus continued 2 -fold higher removal of cholesterol in the feces in $\mathrm{Ldlr}^{-/-} \mathrm{CEHTg}$ mice due to increased FC efflux from macrophages may represent the underlying mechanism for the observed attenuation of dietinduced atherosclerosis in $\mathrm{Ldlr}^{-/-}$CEHTg mice.

The fact that the observed flux of cholesterol from macrophages to bile is in the form of bile acids is consistent with earlier reports demonstrating that while the HDL-associated FC is directly secreted into bile (29), the HDL-CE is efficiently coupled to bile acid synthesis (30).

Several enzymes can catalyze CE hydrolysis, namely HSL, which is primarily an adipose tissue enzyme, and CEL, a bile acid-stimulated pancreatic lipase. Earlier studies have looked for a role of these 2 enzymes in regulating macrophage CE metabolism and, subsequently, atherosclerosis. Transgenic expression of Hsl (8) paradoxically increased atherosclerosis. Since the Hsl transgene was expressed in C57BL/6 mice, a cholic acid-containing diet (cholic acid is a known inhibitor of hepatic bile acid synthesis) was used to induce atherosclerosis and FC efflux from macrophages was not monitored, the results cannot be compared with the present study. Based on the reduced atherosclerosis in Hsl-ApoAIV double transgenic mice compared with Hsl-transgenic mice (for whom atherosclerosis was still higher than ApoAIV transgenics), it was suggested that suboptimal concentration of exogenous FC acceptors was possibly the reason for increased CE in macrophages and increased atherosclerosis in Hsl-transgenic mice (31). Only a direct comparison of the efflux potential of serum from control and Hsl-transgenic mice could confirm this speculation. Using filipin staining, McGookey and Anderson identified an intracellular pool of FC that is associated with cytoplasmic lipid droplets that would not be available for efflux (32). It is tempting to speculate that if Hsl-mediated hydrolysis releases FC to this pool, Hsl overexpression will not result in increased FC efflux. Transgenic expression of Cel, which is a secretory enzyme, also increased atherosclerosis due to increased hydrolysis of ceramide and lysophosphatidyl choline that promotes reesterification and attenuates cholesterol efflux (9). These studies therefore suggest that these 2 "candidate" CE hydrolases do not play a protective role in foam cell formation. On the other hand, the $\mathrm{CEH}$ described in the present study increases FC efflux, reduces net CE accumulation in macrophages, enhances in vivo reverse cholesterol transport, and leads to an attenuation of diet-induced atherosclerosis in Ldlr ${ }^{-/-}$CEHTg mice. Further, this CEH does not share any significant sequence homology with $\mathrm{Hsl}$ or Cel and is a member of the carboxylesterase family. Genome-wide BLAST search of human and mouse genomes shows that while only a single gene coding for $\mathrm{CEH}$ is present on human chromosome 16 (CES1), the corresponding mouse chromosome 8 has at least 9 genes with greater than $95 \%$ homology to $\mathrm{CEH}$. Although the enzymes coded by all these murine genes have not been characterized and their substrate specificities are not yet established, it is tempting to speculate that this redundancy may contribute in part to the inherent protection of mice from atherosclerosis. Future knock- down studies will establish the identity of one or more of these carboxylesterases in mice as bona fide murine macrophage CEH.

Earliest attempts to reduce CE accumulation in macrophages targeted the Acat-mediated FC reesterification step. Targeted disruption of Acat-1, however, led to massive xanthomatosis with little effect on atherosclerosis (33). Acat-1 deficiency also caused marked qualitative differences in atherosclerotic lesions including a lack of CE and markedly decreased macrophage content in advanced lesions due to progressive cell death from FC toxicity (34). Dove et al. showed that although Acat-1 deficiency increases cellular FC, it disrupts cholesterol efflux leading to FC-mediated toxicity (35). The data presented here shows that FC generated by increased hydrolysis of intracellular CE by CEH overexpression not only enhances FC efflux but also reduces macrophage cell death in the lesions (Figure 6). Cellular mechanisms to prevent FC toxicity include Acat-mediated FC esterification and cellular FC efflux (36). Thus strategies targeting Acat- 1 inhibition as the means to reduce cellular CE content eliminate one of the major mechanisms for reducing FC toxicity. On the other hand, enhancing CEH-mediated hydrolysis of intracellular CE maintains both these pathways intact and functional and thereby does not lead to FC accumulation, as shown here and by our earlier data (13, 14). Thus a fundamental difference exists in achieving the goal of reduced cellular CE levels by Acat- 1 inhibition versus $\mathrm{CEH}$ overexpression. The FC generated by $\mathrm{CEH}$-mediated hydrolysis has 2 fates, namely reesterification by Acat-1 or efflux to extracellular acceptors. Thus while in Acat-1-null cells excess FC cannot be reesterified, resulting in cellular toxicity and cell death, in $\mathrm{CEH}$ overexpressing cells, FC released by $\mathrm{CEH}$-mediated hydrolysis is either effluxed or reesterified by Acat-1, resulting in no FC buildup and thus no toxicity or cell death as shown here with highly cellular lesions in Ldlr ${ }^{-/}$CEHTg mice (Figures 5 and 6). It should be pointed out that antisense oligonucleotide-mediated inhibition of Acat-2, the isoform present in liver and intestine and not in macrophages, does reduce atherosclerosis (37), but this effect is due to changes in the plasma lipoprotein profile and has little to do with macrophage CE metabolism.

In conclusion, we have demonstrated for what we believe to be the first time that macrophage-specific expression of $\mathrm{CEH}$ attenuates atherosclerosis and leads to reduced accumulation of CE in the vessel wall. Our studies also indicate that $\mathrm{CEH}$ inhibits atherosclerosis by increasing FC efflux from macrophages and thereby promoting reverse cholesterol transport in vivo and increasing the removal of cholesterol from the body as bile acids. These studies thus provide the much needed "proof of principle" that reducing $\mathrm{CE}$ accumulation in macrophage foam cells by enhancing hydrolysis is antiatherogenic and identify macrophage $\mathrm{CEH}$ as a potentially novel target to attenuate atherogenesis.

\section{Methods}

Production and screening of transgenic mice. All animal protocols were approved by the Institutional Animal Care and Use Committee at the Virginia Commonwealth University. The human scavenger receptor (SR-A) promoter/ enhancer was used to drive macrophage-specific expression of human $\mathrm{CEH}$ in mice. The plasmid pAL1 containing the SR-A promoter/enhancer and all the necessary elements for chimeric transgene construction (poly linker region and heterologous intron) was obtained from Christopher Glass (UCSD, La Jolla, California, USA). Full-length human CEH cDNA ( 2 kb) with an inframe stop codon was cloned into the EcoRI site in the polylinker region of the plasmid pAL1. The sequence of the chimeric transgene 
was confirmed by sequencing. The SRA-CEH chimeric transgene was separated from the vector sequence by digestion with NotI and XhoI, purified by agarose gel electrophoresis, and injected into the pronuclei of fertilized mouse eggs obtained from superovulated female mice (C57BL/6 or Balb-c/ C57BL/6 hybrids). The injected eggs were surgically transferred to oviducts of surrogate females. Offspring were screened for integration of the transgene by PCR amplification of the tail DNA with the upstream primer sequence 5'-ATGGCAAAGTGCTGGGGAAGTTC-3' and the downstream primer sequence $5^{\prime}$-TTCACCAGAACAGAAGTGAGGGC-3' to yield a 681bp product corresponding to the nucleotide sequence between residues 133 and 792 of human macrophage $\mathrm{CEH}$ cDNA. The transgenic founder lines were maintained and propagated. To evaluate the role of $\mathrm{CEH}$ in the development of atherosclerosis, $\mathrm{CEH}$-transgenic mice were crossed with atherosclerosis-susceptible Ldlr $/-$ mice that had a C57BL/6 background (The Jackson Laboratory). All animals were genotyped for CEH and LDLR. Two upstream primers were used, 5'-AGAGCAGCCGATTGTCTGTTGTG-3' (83-105 bp of the Neo cassette) and 5'-TGTGTGATGGAGACCGAGATTGC-3' (380-402 bp of mouse LDLR), along with one downstream primer, 5'-CAGTGCTCCTCATCTGACTTGTC-3' (673-695 bp of mouse LDLR), to yield a single PCR product of $500 \mathrm{bp}$ and $316 \mathrm{bp}$ with $\mathrm{Ldll}^{-/-}$and $\mathrm{LDLR}^{+/+}$ genotypes, respectively. Both products were obtained with heterozygous $\mathrm{LDLR}^{+/-}$mice. Littermates with or without CEHTg in $\mathrm{Ldll}^{-/-}$background ( $\mathrm{Ldlr}^{-/-} \mathrm{CEHTg}$ and $\mathrm{Ldlr}^{-/-}$mice) were included in the studies.

Measurement of $\mathrm{CEH}$ expression. Mice were injected intraperitoneally with $4 \%$ thioglycollate solution, and macrophages were harvested 4 days later by lavage of the peritoneal cavity. The peritoneal macrophages were resuspended in RPMI containing 10\% fetal bovine serum. Total RNA from adherent cells was extracted after 24 hours using RNeasy kit (QIAGEN). Liver, lung, spleen, kidney, and heart were also harvested and total RNA extracted using TriReagent (Molecular Research Center). The expression profile of CEH was determined by real time PCR, and CEH mRNA copy number in each tissue per unit of total RNA was calculated using a standard curve as described in ref. 38. CEH activity in adherent macrophages was determined using the radiometric assay (39).

Total plasma cholesterol and cholesterol distribution among plasma lipoproteins. A modified column lipoprotein profile method was used (40). Whole plasma aliquots frozen and stored at $-80^{\circ} \mathrm{C}$ were thawed at $4^{\circ} \mathrm{C}$. Total plasma cholesterol concentration was determined by a microenzymatic method. Approximately $20 \mu \mathrm{g}$ of cholesterol was injected onto an FPLC system (Superose 6 HR 10/30 column; Amersham Biosciences) with online mixing of the column effluent with enzymatic reagent (Cholesterol Liquid Stable; Thermo Electron) for acquiring lipoprotein cholesterol profiles. The output generated is in millivolts. The data were acquired on a personal computer running ChromPerfect Spirit chromatography software (Justice Software). The system was optimized so that the area under the profiles was proportional to the cholesterol mass. Area percent in each lipoprotein fraction, VLDL, LDL, and HDL was applied to total plasma cholesterol to calculate cholesterol concentration in the lipoprotein fractions.

Quantitative atherosclerosis analyses. Male and female littermates with or without CEHTg in Ldlr ${ }^{-/-}$background (Ldlr ${ }^{-/}$CEHTg and $\mathrm{Ldll}^{-/}$) were included in the study at 10 weeks of age. The total number of animals in each group was therefore determined by the availability of the correct genotype and gender within a litter. Mice were fed a Western-type high-fat, highcholesterol diet (TD88137; Harlan Teklad), which contained 21\% fat, $0.15 \%$ cholesterol, and $19.5 \%$ casein by weight with no sodium cholate. Animals were weighed every 2 weeks, and after 16 weeks nonfasting plasma was collected. The aorta was dissected from the heart to the iliac bifurcation, cleaned of any surrounding tissue, opened longitudinally, pinned on black wax, and fixed for 24 hours in $10 \%$ buffered formalin. The fixed aortae were imaged on a black background using a Canon digital camera fitted with a 60-mm, f/2.8 Macro Lens. Total area and the area occupied by the lesions in the aortic arch and thoracic and abdominal aortae was determined using BIOQUANT Image Analysis 98 software. The person quantifying the area occupied by lesions was blinded to the identity of the images. Extreme care was taken to ensure that any residual adventitial fat that appeared translucent on the images was not included in the area occupied by the lesions that were dense and opaque. For determination of EC mass of the lesions, the aorta was cut into 2 pieces, the aortic arch and the thoracic and abdominal aortae, along the line shown in Figure 3, and FC and EC masses were determined in these 2 pieces as previously described (41). Briefly, lipids were extracted in 2:1 chloroform/methanol with $5 \alpha$ cholestane added as an internal standard, and FC and total cholesterol (after saponification) were measured by gas-liquid chromatography. EC mass was determined as the difference between total cholesterol and FC. The delipidated tissue protein was then digested and dissolved in $1 \mathrm{~N} \mathrm{NaOH}$, and total protein was determined (42). Data ( $\mu$ g cholesterol/mg total protein) are expressed as mean \pm SD for 6 animals per genotype.

Morphological analyses of the lesions. Hearts from mice fed a high-fat, highcholesterol diet were fixed in buffered formalin, paraffin embedded, and sectioned. Once the aortic sinus was visible, $3-4$ serial sections ( $4 \mu \mathrm{m}$ thick) were transferred to numbered slides. Consequently, numbered slides were then stained with Masson's Trichrome stain and H\&E and immunostained with antibody to macrophage marker MOMA-2 (43) or stained to determine the TUNEL-positive macrophages using the In Situ Cell Death Detection Kit, POD (Roche). Images were acquired using an Olympus inverted microscope fitted with a digital camera at the magnifications indicated in the figure legends. Images of the H\&E-stained sections were used to determine the total acellular area of the lesion using the BIOQUANT software. This area is expressed as a percentage of the total lesion area. Total number of macrophages were counted in 3 fields $(300 \times 420 \mu \mathrm{m})$ per section, and the mean $\pm \mathrm{SD}$ was determined for 5 mice per genotype and 3 lesions per animal. Total number of nuclei as well as TUNEL-positive nuclei were determined from serial sections, and data are expressed as percent TUNEL-positive nuclei.

Cholesterol efflux from peritoneal macrophages. Thioglycolate-elicited macrophages from chow-fed animals were plated in 12-well tissue culture plates in RPMI supplemented with $10 \%$ FBS. Nonadherent cells were removed after 24 hours, and the intracellular FC and EC pools were labeled with $\left[{ }^{3} \mathrm{H}\right]$-cholesterol and FC for 24 hours. Similar distributions of radioactivity in FC $\left(54.3 \% \pm 5.5 \%\right.$ in $\mathrm{Ldll}^{-/}$versus $59.7 \% \pm 2.1 \%$ in $\left.\mathrm{Ldlr}^{-/-\mathrm{CEHTg}}\right)$ and $\mathrm{EC}$ $\left(45.7 \% \pm 5.5 \%\right.$ in $\mathrm{Ldll}^{-/-}$versus $40.3 \% \pm 2.1 \%$ in $\left.\mathrm{Ldll}^{-/} \mathrm{CEHTg}\right)$ were observed in macrophages from either genotype following 24-hour equilibration. FC efflux to ApoA1, HDL, and 10\% FBS was determined as described previously (14). Cholesterol efflux experiments were performed in duplicate, and the data (\% efflux) are expressed as mean \pm SD for 3 animals per genotype.

Gene expression in peritoneal macrophages. Thioglycollate-elicited macro-

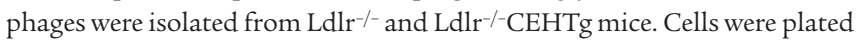
in $60-\mathrm{mm}$ tissue culture dishes and nonadherent cells removed after 24 hours. One set of cells was loaded using AcLDL $(25 \mathrm{mg} / \mathrm{ml})$ for 48 hours. Total RNA was extracted from nonloaded and loaded cells using RNeasy Kit (QIAGEN) and expression of Abca1, Abcg1, Cd36, Msr1, Scarb1, and CEH determined by real time RT-PCR as described previously (35). The level of expression for each gene in AcLDL-loaded cells was normalized to the corresponding levels in nonloaded cells.

Measurement of cholesterol from macrophages to bile and feces in vivo. For $\left[{ }^{3} \mathrm{H}\right]$-cholesterol labeling and cholesterol loading, thioglycollate-elicited macrophages were isolated from $\mathrm{Ldll}^{-/-}$and $\mathrm{Ldlr}^{-/-} \mathrm{CEHTg}$ mice. Cells from 4 mice were pooled and plated in $100-\mathrm{mm}$ petri dishes. After 4 hours, nonadherent cells were removed and adherent cells were radiolabeled with $5 \mu \mathrm{Ci} / \mathrm{ml}\left[{ }^{3} \mathrm{H}\right]$-cholesterol and cholesterol enriched with $50 \mu \mathrm{g} / \mathrm{ml}$ of 
AcLDL for 24 hours (18). These foam cells were equilibrated in serum-free medium with $0.2 \%$ bovine serum albumin for 24 hours. Cells were washed twice with MEM containing no calcium or magnesium and then detached using the same medium supplemented with 20 mM EDTA. Cells were collected by repeated pipeting, spun down, and resuspended in RPMI medium immediately before use. The distribution of $\left[{ }^{3} \mathrm{H}\right]$-cholesterol measured by TLC (64\%-68\% in CE) and cholesterol mass measured by GLC (63\%-67\% in $\mathrm{CE})$ between FC and CE were very similar between macrophages from both genotypes. On average, the cell suspension contained $2 \times 10^{6} \mathrm{cells} / \mathrm{ml}$ at $1.5 \times 10^{6} \mathrm{dpm} / \mathrm{ml}$ and total cellular cholesterol of $4.3 \mu \mathrm{g}$.

For in vivo studies, wild-type C57BL/6 mice (The Jackson Laboratory) maintained on chow diet were injected intraperitoneally with cholesterolloaded and $\left[{ }^{3} \mathrm{H}\right]$-cholesterol-labeled foam cells from either $\mathrm{Ldll}^{-} /$or $\mathrm{Ldll}^{-/}$ CEHTg mice $(0.5 \mathrm{ml})$ and transferred to metabolic cages. Feces were collected from 0 to 48 hours and stored at $-20^{\circ} \mathrm{C}$ prior to lipid extraction. At 48 hours, mice were euthanized and gall bladder bile was collected.

For lipid extractions, biliary cholesterol and bile acids were extracted from gall bladder bile as described (44). Total feces were vacuum dried and powdered, and fecal cholesterol as well as bile acid were extracted as described by Batta et al. (45). Values are expressed as a percent of total injected $\left[{ }^{3} \mathrm{H}\right]$-cholesterol as described by Moore et al. (46).

Statistics. Results were analyzed by 2 -tailed Student's $t$ test with the use of GraphPad Prism version 4 software, which tests for normality before run- ning the Student's $t$ test. While statistical significance for all comparisons was assigned at $P<0.05$, the individual $P$ values are included in the text.

\section{Acknowledgments}

This work was supported by research grant (HL-069946) from National Heart, Lung, and Blood Institute to S. Ghosh. The authors thank Christopher Glass for providing the pAL1 vector. The transgenic mice were developed in the Institutional Massey Cancer Center Transgenic/Knock-out Mouse Core facility supported in part by a grant (P30 CA16059) from NCI. We thank Daniel Radar, Jeffrey Billheimer, and George Rothblat for providing the protocol used for measuring in vivo reverse cholesterol transport using mouse peritoneal macrophages and Rajendra Tangirala for advice on the morphological analyses of sinus lesions.

Received for publication September 27, 2006, and accepted in revised form June 26, 2007.

Address correspondence to: Shobha Ghosh, Department of Internal Medicine, Division of Pulmonary and Critical Care, Virginia Commonwealth University, Room 8-047, Sanger Hall, 1101 E. Marshall Street, Richmond, Virginia 23298-0050, USA. Phone: (804) 827-1012; Fax: (804) 827-1782; E-mail: Shobha@vcu.edu.
1. Tabas, I. 2000. Cholesterol and phospholipids metabolism in macrophages. Biochim. Biopbys. Acta. 1529:164-174.

2. Rothblat, G.H., Llera-Moya, M.D.L., Favari, E., Yancey, P.G., and Kellner-Wiebel, G. 2002. Cellular cholesterol flux studies: methodological considerations. Atherosclerosis. 163:1-8.

3. Goldberg, D.I., and Khoo, J.C. 1990. Stimulation of neutral cholesteryl ester hydrolase by cAMP in P388D1 macrophages. Biochim. Biophys. Acta. 1042:132-137.

4. Small, C.A., Goodacre, J.A., and Yeaman, S.J. 1989. Hormone sensitive lipase is responsible for the neutral cholesteryl ester hydrolase activity in macrophages. FEBS Lett. 247:205-208.

5. Khoo, J.C., Reue, K., Steinberg, D., and Schotz, M.C. 1993. Expression of hormone-sensitive lipase mRNA in macrophages. J. Lipid Res. 34:1969-1974.

6. Osuga, J., et al. 2000. Targeted disruption of hormone-sensitive lipase results in male sterility and adipocyte hypertrophy, but not in obesity. Proc. Natl. Acad. Sci. U. S. A. 97:787-792.

7. Contreras, J.A. 2002. Hormone sensitive lipase is not required for cholesteryl ester hydrolysis in macrophages. Biochem. Biophys. Res. Comm. 292:900-903.

8. Escary, J.L., et al. 1999. Paradoxical effect on atherosclerosis of hormone-sensitive lipase overexpression in macrophages. J. Lipid Res. 40:397-404.

9. Li, F., and Hui, D.Y. 1997. Modified low-density lipoprotein enhances the secretion of bile saltstimulated cholesterol esterase by human monocyte-macrophages. Species-specific difference in macrophage cholesteryl ester hydrolase. J. Biol. Chem. 272:28666-28671.

10. Kodvawala, A., Ghering, A.B., Davidson, W.S., and Hui, D.Y. 2005. Carboxyl ester lipase expression in macrophages increases cholesteryl ester accumulation and promotes atherosclerosis. J. Biol. Chem. 280:38592-38598.

11. Ghosh, S. 2000. Cholesteryl ester hydrolase in human monocyte/macrophage: cloning, sequencing and expression of full-length cDNA. Physiol. Genomics. 2:1-8.

12. Zhao, B., Fisher, B.J., St. Clair, R.W., Rudel, L.L., and Ghosh, S. 2005. Redistribution of macrophage cholesteryl ester hydrolase from cytoplasm to lipid droplets upon lipid loading. J. Lipid Res. 46:2114-2121.
13. Ghosh, S., St. Clair, R.W., and Rudel, L.L. 2003. Mobilization of cytoplasmic CE droplets by overexpression of human macrophage cholesteryl ester hydrolase. J. Lipid Res. 44:1833-1840.

14. Zhao, B., Song, J., St. Clair, R.W., and Ghosh, S. 2007. Stable over-expression of human macrophage cholesteryl ester hydrolase $(\mathrm{CEH})$ results in enhanced free cholesterol efflux from human THP1-macrophages. Am. J. Physiol. Cell Physiol. 292:C405-C412.

15. Horvai, A., et al. 1995. Scavenger receptor A gene regulatory elements target gene expression to macrophages and to foam cells of atherosclerotic lesions. Proc. Natl. Acad. Sci. U. S. A. 92:5391-5395.

16. Veniant, M.M., et al. 2000. Defining the atherogenicity of large and small lipoproteins containing apolipoprotein B100. J. Clin. Invest. 106:1501-1510.

17. Asztalos, B.F., et al. 2005. Differential effects of HDL subpopulations on cellular ABCA1- and SR-BI-mediated cholesterol efflux. J. Lipid Res. 46:2246-2253.

18. Zhang, Y., et al. 2003. Over-expression of Apolipoprotein A-I promotes reverse cholesterol transport from macrophages to feces in vivo. Circulation. 108:661-663.

19. Brown, M.S., Ho, Y.K., and Goldstein, J.L. 1980. The cholesteryl ester cycle in macrophage foam cells. Continual hydrolysis and re-esterification of cytoplasmic cholesteryl esters. J. Biol. Chem. 255:9344-9352.

20. Khoo, J.C., Mahoney, E.M., and Steinberg, D. 1981. Neutral cholesterol esterase activity in macrophages and its enhancement by cAMP-dependent protein kinase. J. Biol. Chem. 256:12659-12661.

21. Ishii, I., et al. 1992. Beta-VLDL-induced cholesterol ester deposition in macrophages may be regulated by neutral cholesterol esterase activity. Arterioscler. Thromb. 12:1139-1145.

22. Yancey, P.G., and St. Clair, R.W. 1994. Mechanism of the defect in cholesteryl ester clearance from macrophages of atherosclerosis-susceptible White Carneau pigeons. J. Lipid Res. 35:2114-2129.

23. Hakamata, H., et al. 1994. Species difference in cholesteryl ester cycle and HDL-induced cholesterol efflux from macrophage foam cells. Arterioscler. Thromb. 14:1860-1865.

24. Bjorkhem, I., et al. 1994. Atherosclerosis and sterol 27-hydroxylase: evidence for a role of this enzyme in elimination of cholesterol from human macrophages. Proc. Natl. Acad. Sci. U. S. A. 91:8592-8596.

25. Zhang, W.Y., Gaynor, P.M., and Kruth, H.S. 1996. Apolipoprotein E produced by human monocytederived macrophages mediates cholesterol efflux that occurs in the absence of added cholesterol acceptors. J. Biol. Chem. 271:28641-28646.

26. Yancey, P.G., et al. 2003. Importance of different pathways of cellular cholesterol efflux. Arterioscler. Thromb. Vasc. Biol. 23:712-719.

27. Wang, N., Lan, D., Chen, W., Matsuura, F., and Tall, A.R. 2004. ATP-binding cassette transporters G1 and G4 mediate cellular cholesterol efflux to highdensity lipoproteins. Proc. Natl. Acad. Sci. U. S. A. 101:9774-9779.

28. Mazzone, T., Krishna, M., and Lange, Y. 1995. Progesterone blocks intracellular translocation of free cholesterol derived from cholesteryl ester in macrophages. J. Lipid Res. 36:544-551.

29. Schwartz, C.C., Halloran, L.G., Vlahcevic, Z.R. Gregory, D.H., and Swell, L. 1978. Preferential utilization of free cholesterol from high-density lipoproteins for biliary cholesterol secretion in man. Science. 200:62-64.

30. Pieters, M.N., et al. 1991. Selective uptake of cholesteryl esters from apolipoprotein-E-free high-density lipoproteins by rat parenchymal cells in vivo is efficiently coupled to bile acid synthesis. Biochem. J. 280:359-365.

31. Choy, H.A., Wang, X.P., and Schotz, M.C. 2003. Reduced atherosclerosis in hormone-sensitive lipase transgenic mice over-expressing cholesterol acceptors. Biochim. Biophys. Acta. 1634:76-85.

32. McGookey, D.J., and Anderson, R.G.W. 1983. Morphological characterization of the cholesteryl ester cycle in cultured mouse macrophage foam cells. J. Cell Biol. 97:1156-1168.

33. Yagyu, H., et al. 2000. Absence of ACAT-1 attenuates atherosclerosis but causes dry eye and cutaneous xanthomatosis in mice with congenital hyperlipidemia. J. Biol. Chem. 275:21324-21330.

34. Accad, M., et al. 2000. Massive xanthomatosis and altered composition of atherosclerotic lesions in hyperlipidemic mice lacking acyl CoA:cholesterol acyltransferase 1. J. Clin. Invest. 105:711-719.

35. Dove, D.E., et al. 2005. ACAT1 deficiency disrupts cholesterol efflux and alters cellular morphol- 
ogy in macrophages. Arterioscler. Thromb. Vasc. Biol. 25:128-134.

36. Feng, B., et al. 2003. The endoplasmic reticulum is the site of cholesterol-induced cytotoxicity in macrophages. Nat. Cell Biol. 5:781-792.

37. Bell, T.A., 3rd, et al. 2006. Liver-specific inhibition of acyl-coenzyme a:cholesterol acyltransferase 2 with antisense oligonucleotides limits atherosclerosis development in apolipoprotein B100-only low-density lipoprotein receptor ${ }^{-/-}$mice. Arterioscler. Thromb. Vasc. Biol. 26:1814-1820.

38. Zhao, B., Natarajan, R., and Ghosh, S. 2005. Human liver cholesteryl ester hydrolase: cloning, molecular characterization, and role in cellular cholesterol homeostasis. Physiol. Genomics. 23:304-310.

39. Ghosh, S., and Grogan, W.M. 1989. Activation of rat liver cholesterol ester hydrolase by cAMPdependent protein kinase and protein kinase C. Lipids. 24:733-736.

40. Garber, D.W., Kulkarni, K.R., and Anantharamaiah, G.M. 2000. A sensitive and convenient method for lipoprotein profile analysis of individual mouse plasma samples. J. Lipid Res. 41:1020-1026.

41. Rudel, L.L., Kelley, K., Sawyer, J.K., Shah, R., and Wilson, M.D. 1998. Dietary monounsaturated fatty acids promote aortic atherosclerosis in LDL receptor null, human apoB100 overexpressing transgenic mice. Arterioscler. Thromb. Vasc. Biol. 18:1818-1827.

42. Lowry, O.J., Rosebrough, N.J., Farr, A.L., and Randall, R.J. 1951. Protein measurement with the Folin phenol reagent. J. Biol. Chem. 193:265-275.
43. Kusunoki, J., et al. 2001. Acyl-CoA:cholesterol acyltransferase inhibition reduces atherosclerosis in apolipoprotein E deficient mice. Circulation. 103:2604-2609.

44. Ren, S., et al. 2004. Overexpression of cholesterol transporter StAR increases in vivo rates of bile acid synthesis in the rat and mouse. Hepatology. 40:910-917.

45. Batta, A.K., et al. 1999. Highly simplified method for gas-liquid chromatographic quantitation of bile acids and sterols in human stool. J. Lipid Res. 40:1148-1154.

46. Moore, R.E., et al. 2005. Increased atherosclerosis in mice lacking apolipoprotein A-I attributable to both impaired reverse cholesterol transport and increased inflammation. Circ. Res. 97:763-771. 